\title{
The Predicament and Improvement Strategy of Primary and Middle School Educational Punishment in China
}

\author{
Li PuHua ${ }^{1, a}$ \\ 1 The General Office, Jiangsu University, Zhenjiang, Jiangsu, China \\ alipuhua@126.com
}

\begin{abstract}
The Educational Punishment Rules for Primary and Secondary Education (Trial Implementation) promulgated in 2020 has played a vital role in standardizing and improving the educational punishment behavior of primary and secondary education. As the main body of educational punishment, teachers bear the dual identity of judgment and implementation of educational punishment, and are vulnerable to personal factors in the process of educational punishment, which brings harm to students' body and mind. At present, the dilemma of implementing educational punishment in China includes the vague definition of the implementation standard of educational punishment, the need to improve the level of teachers' professional development, the imperfect coordination of educational punishment, and the difficulty to comprehensively measure the effect of implementing educational punishment. Therefore, we should clarify the criteria of educational punishment, standardize the educational punishment of school education, promote the development of teachers, improve the level of educational punishment, improve family responsibility, coordinate family educational punishment, multicoordinate education, and optimize social educational punishment.it is committed to building a pluralistic educational punishment system coordinated, coordinated and supervised by schools, families and society, and constantly improving the systematicness and practical effectiveness of educational punishment.
\end{abstract}

Keywords: Punishment, Educational Punishment, Power of Educational Punishment

\section{中国中小学教育惩戒困境与改进策略}

李普华 $1, \mathrm{a}$

\begin{abstract}
${ }^{1}$ 校长办公室, 江苏大学, 镇江, 江苏, 中国
alipuhua@126.com

\section{摘要}

2020 年颁布的《中小学教育惩戒规则 (试行)》对于规范和完善中小学教育惩戒行为起到了至关重要的作用。 但作为教育惩戒主体的教师，承担了教育惩戒“判定”和教育惩戒“实施”的双重身份，教育惩戒过程中容易受个 人因素影响给学生身心带来危害。当前, 我国实施教育惩戒的困境包括教育惩戒实施标准的界定较为模糊、教 师专业发展水平有待提高、教育惩戒的协同不够完善、教育惩戒实施效果的评估难以全面衡量。为此, 我们应 明确惩戒标准, 规范学校教育惩戒, 推动教师发展, 提升教育惩戒水平, 完善家庭责任, 协同家庭教育惩戒, 多元协同育人，优化社会教育惩戒，致力于构建由学校、家庭、社会相互协调、配合、监督的多元教育惩戒体 系，不断提升教育惩戒的系统性和实际成效。
\end{abstract}

关键词：惩戒，教育惩戒，教育惩戒权 


\section{1.前言}

教育惩戒作为教育手段的组成部分, 伴随着教育 活动的产生而产生。随着社会民主法治的逐步发展和 完善, 对教育惩戒的认识得到了进一步深化。近年教 师体罚学生、辱骂学生等起诉教师侵权案件频发, 使 得教育惩戒行为立法的紧迫性和必要性进一步增强。 《中小学教育惩戒规则（试行)》(中华人民共和国教 育部令第 49 号) 作为规范和完善中小学教育惩戒的 纲领性文件, 对规范和完善教育惩戒行为具有重要作 用。但教育惩戒的实施主体教师具有教育惩戒“判定” 和教育惩戒“实施”的双重身份，容易因实施惩戒的主 体情绪变化, 造成对学生违规行为判定标准或者实施 方式的不同。为避免因教育惩戒主体“双重身份”带来 的惩戒不当造成的学生伤害, 我们应着力推动构建多 元教育惩戒体系，提升教育惩戒的系统性。

《辞海》对于惩罚定义为, 惩罚即惩戒, 惩治过 错, 警戒未来。[1]“惩罚在道德上的正当性在于它的 效果——即对于预防犯罪和使罪犯重新适应社会最 初的贡献。惩罚从实质上讲是朝前看的: 它关心我们 对于社会, 包括罪犯, 可能做的将来的善事。”[2]《教 育大辞典》将教育惩罚界定为“对个体或者集体的不 良行为给予否定或批评处分, 旨在制止某种行为的发 生, 与奖励相对, 为学校德育采取的一种教育方法, 有利于学生分辨是非善恶, 削弱受罚行为动机, 达到 改正的目的, 也有利于维护校规校级。”[3]李秀娟认 为: “教育惩戒是指通过给学生施加某种影响, 使其感 到痛苦或差耻, 激发其悔意, 从而达到矫正的目的。”[4] 从以上对教育惩罚和教育惩戒内涵的界定来看, 我国 的教育惩戒是为了戒除不良的行为而采取的一种合 乎道德的手段。2020 年 12 月, 教育部颁布了《中小 学教育惩戒规则 (试行)》, 明确了教育惩戒是指学校、 教师基于教育目的, 对违规违纪学生进行管理、训导 或者以规定方式予以矫治, 促使学生引以为戒、认识 和改正错误的教育行为。[5]同时该文件还进一步明确 了教师实施教育惩戒的适用范围、实施原则、实施形 式和申诉监督等内容为教师教育惩戒提供了遵循。但 从教育惩戒行为的属性来看, 教育惩戒作为一种手段, 本身不含有好、坏的性质, 只因惩戒的方式、效果不 同而造成教育惩戒的恰当与否; 教育惩戒不仅是一种 教育管理手段, 更是一种教育教学行为, 具有教育艺 术性。教育惩戒必须建立在一种表象技术上, 只有当 教育惩戒顺其自然的成为教育的一部分, 教育惩戒才 是成功的; 教育惩戒的对象是学生过去做的不良行为, 而不是学生本身, 故而教育惩戒不是一种人身惩戒, 更不是一种报复行为; 教育惩戒是为了减少或者避免 学生不良行为的发生, 教育惩戒的效果相对具有滞后 性。

\section{2. 我国实施教育惩戒的困境}

当前大家对教育惩戒认识不一, 教育法律法规对 教育惩戒判定的界限较为模糊, 教育惩戒的协同不够 完善, 教育惩戒实施效果的评估难以全面衡量, 使得
教育惩戒实施后的效果受到较大影响。

\section{1. 教育惩戒实施标准的界定较为模糊}

我国《中小学教育惩戒规则 (试行)》第七条明确 了“扰乱课堂秩序、学校教育教学秩序”等情况可以实 施教育惩戒，第八条、第九条、第十条、第十一条明 确了违规违纪情节较为轻微的、情节较重的、影响恶 劣的如何实施教育惩戒。[6]但对于学生违规违纪行为 轻微、较重、严重等性质的判定尚未明确, 导致教师 实施教育惩戒行为的判定标准较为模糊。近日, 厦门 翔安巷南中学初二学生, 因违反纪律遭到年级组长罚 站一节课, 在学校楼梯徘徊后于当天晚上坠楼。[7]中 小学生正处于身心发育的关键时期, 教育惩戒标准由 教师个人判定，容易导致教师惩戒实施的偏差，进而 影响学生的身心健康。比如在教学管理中对于“调皮 学生”的认识也是仁者见仁: 有的教师认为“调皮学生” 足够的聪明、有灵性, 要多加以正确的引导和鼓励; 但也有教师认为“调皮学生”在扰乱课堂教学秩序, 可 以按照规定给予教育惩戒。受限于惩戒实施的界限较 为模糊, 对于如何实施教育惩戒需要更多的智慧, 为 避免教育惩戒带有随意性, 教育惩戒某种程度已成为 教师想用却不敢用的“教育方式”。

\section{2. 教师专业发展水平有待提高}

教育现代化意味着未来教育发展的趋势和结果, 是教育系统整体性的现代化变革, 教育系统的每个要 素都需要适应现代化的教育发展, 并随之变化调整, 才能适应现代化教育系统的需要, 与之形成共振, 充 分发挥自己的作用, 最终使相关要素在系统中很好地 适应和发展, 教师要素也不例外。[8]随着社会进步和 教育民主化运动的开展, 许多国家宣布体罚为非法活 动, 我国于 1952 年 4 月由原国家教委发布了有关禁 止对学生体罚或变相体罚的指示。但因为一些教师在 思想上认为体罚学生也是教育惩戒的一种形式, 造成 了主观认识的偏差。除了思想认识上存在着差距外, 由于近年教师队伍不断扩大, 师范生教育与教师从业 资格政策的不断调整, 致使我国教师专业发展水平有 待提高。尤其是部分青年教师从研究生毕业后, 缺乏 足够的教育教学基本技能训练和师德师风培训, 致使 从业后在师德师风、教学技能上不能满足实际教学需 求。受限于公办学校和私立学校、有编制教师和无编 制教师等身份差异, 东西部经济发展不均衡, 教师工 资待遇水平普遍低于公务员收入水平, 导致部分中小 学教师对本职工作缺乏热情、事业心和责任感, 民主 意识甚差, 不以平等的态度对待学生, 不懂得教育艺 术, 甚至对学生缺乏最起码的爱心、耐心和服务意识, “一切为了孩子，为了一切孩子，为了孩子的一切”在 实际工作中成了一句空话。教师专业发展水平严重制 约了教育惩戒的有效实施，可以说，提升教师专业发 展水平是有效实施教育惩戒的内在要求, 也是提升教 育教学质量的关键路径。 


\section{3. 教育惩戒的协同不够完善}

完整的全面教育既包括学校教育、家庭教育, 也 包括社会教育。教育的主体是多元的, 在基于培养人 的教育活动中实施教育惩戒的主体也应是多元的, 且 应该相互贯通。对于学生在学校犯的一般性错误, 应 由教师在学校实施相应的教育惩戒, 如果由父母在课 后代替教师来实施惩戒, 教育惩戒的及时性就无法体 现。同样, 对于学生较为严重的违规行为, 仅仅由教 师在学校进行批评教育也无法达到入脑入心。学校教 育、家庭教育、社会教育的开展形式不同、场所不同， 具体实施教育惩戒的方式也有所差异, 唯有在教师、 家长、社会等教育惩戒主体之间形成有效的沟通, 达 成一种合理的契约, 共同对学生的错误实施惩戒, 使 得他们之间相互监督、相互补充, 形成对学生的立体 式教育、全面教育。

\section{4. 教育惩戒实施效果的评估难以全面衡量}

基于教育惩戒向善的效果, 教育惩戒被认为教育 中必不可少的一部分。“惩罚不得不通过它的实际效 果而证明它是合理的。这种效果对于社会来说, 是用 不着把残忍的惩罚合法化就能维护秩序; 对于罪犯来 说, 是对他进行遏制并促进他的改造。要把这种防卫 手段化成改造工具, 需要最高的智慧和人生。”[9]但 从教师实施教育惩戒的效果来看, 多是教师批评教育 了学生, 或对学生进行了严格的问责管理, 让课堂班 级管理变得更加和谐; 但对于个性发展的学生而言, 广大教师缺少了鼓励、关爱, 使得学生在心理上造成 了挫败感，自信心逐渐下降或者师生之间产生了隔阂， 师生关系越来越紧张, 惩戒应有的向善效果没有得到 体现, 教育惩戒成了一种缺乏关爱的刚性教育管理措 施。

\section{3. 实施教育惩戒的改进策略}

新修订的《未成年人保护法》第一百一十三条规 定“对违法犯罪的未成年人, 实行教育、感化、挽救的 方针, 坚持教育为主、惩罚为辅的原则”。[10]教师公 开惩戒学生的意义不在于对受罚者本人的差辱, 而在 于进行一种表象的制造与传播, 也就是说惩罚并不是 在利用通过给受罚者肉体带来的那种“痛苦”的实际 感觉,而是需要通过那种惩罚, 让受罚者产生一种痛苦、 不愉快的观念, 以及一种“违规就要受到惩罚”的关联, 从而使这种感觉慢慢地进入到所有的学生的观念中 去。[11]为避免因教育惩戒实施不当带来的危害, 实 施教育惩戒需要学校教育内部与外部的互相配合, 建 构由学校、家长、社会机构组成的多元教育惩戒体系, 有助于提升教育惩戒实施的合法性、公正性、有效性。

\section{1. 明确惩戒标准, 规范学校教育惩戒}

苏联教育家马卡连柯提出要将惩罚与对学生的 尊重联系起来, “凡是需要惩罚的地方, 教师就没有权 利不惩罚。在必须惩罚的情况下, 惩罚不仅是一种权
利，而且是一种义务，也就是说，我深信教师可以用 惩罚或不用惩罚，但是，如果教师的良心、教师的熟 练技术、教师的信念说明他应当惩罚时，他也就没有 权利拒绝使用惩罚。惩罚也和其他的一切方法一样, 也应当用一种自然的、通常的和合乎逻辑的方法来提 出。”[12]教育惩戒作为一种教育手段, 它不是教育的 目的, 更不是教师发泄怨气的途径。教师依法享有惩 戒学生的权利, 但教育惩戒学生不得以侵害学生权利, 甚至牺牲学生合法权益为代价。为此, 我们应建立学 校惩戒机构, 规范教师的惩戒行为, 借助“教育戏剧” 等情景式教学, 帮助学生构建品德发展地图, 指导教 师因人、因地、因事、因时合理实施惩戒, 监督教师 的惩戒行为, 避免教师实施教育惩戒的随意性和主观 性。同时, 依托学校惩戒机构维护学生权益, 使得教 育惩戒学生的权利可以通过正确途径, 避免因教师个 人的主观臆断或者个人喜好, 对某些学生偏袒过度或 者惩戒过度。

\section{2. 推动教师发展，提升教育惩戒水平}

教育现代化目标下，教师需要具备先进的思想理 念，也需要形成广阔的视野、丰富而又熟练的教育技 能, 以及先进的教育手段等。需要教师的人文素养、 专业素养和科研素养等方面发展至更高水平。[13]在 中国, 教师是履行教育教学职责的专业人员, 尤其是 在中小学从事工作的教师具有更强的特殊性和专业 性。教育惩戒只是教育的一种手段, 它不是教育教学 的目的, 教育惩戒的理念应与教育教学的基本理念保 持一致, 要为教育而惩戒, 要为促进学生健康积极、 全面发展而实施惩戒, 这就需要教师不断提升综合素 养和专业发展水平。在实施教育惩戒的时候, 教师更 多的需要运用教学艺术和技巧, 从构建学生最近发展 区和促进学生行为养成的角度出发, 推动学生从内心 里认可并接受教师实施的教育惩戒, 做到“口服心服”, 而不是因为教师的权威而屈服, 最终“口服心不服”。 从教育惩戒本身来说, 如何实施教育惩戒本身就是一 门艺术, 有人认为教师的惩戒是教师的关心，因教师 实施惩戒有效改正了错误的行为而内心愉悦, 并对教 师心存感激, 这也对教师如何提升教育惩戒的科学性 和艺术性提出了更高的挑战。加强教师专业发展不仅 是引进高精尖人提升师资队伍水平，同时也需要不断 加强教师的师德师风和教学素养, 让教育惩戒变成 “有温度”的教学，否则“冷冰冰”的教育惩戒注定是失 败的。而在实施教育惩戒时, 尊重学生的尊严是教师 实施教育惩戒的前提, 应把尊重学生的独立人格和尊 严放在第一位。所谓“学高为师，德高为范”，真正的 教师不仅学养深厚，具备良好的“传道、授业、解惑” 的能力, 还应该具有较高的道德水准, 使自己成为学 生的道德行为准则, 同时也应该具备良好的心理素质, 能够在学生犯错的时候不意气用事, 能够冷静、理智 地实施惩戒; 在教育惩戒实施后, 教师还能够一如既 往的关心和爱护受罚的学生, 不因其一次错误而改变 对他的态度，不使其产生失落感、孤独感。[14] 


\section{3. 完善家庭责任，协同家庭教育惩戒}

了解学生的人莫过于家庭, 最爱学生的人是父母, 实施有效的惩戒需要家庭的积极配合, 以形成教育协 同合力。过去的家庭教育往往自扫门前雪, 大家互不 干涉, 由于家庭教育观念相对落后、体系不够完善等, 家长对学生的教育惩戒未能有效实施。, 教育惩戒的 真正落实需获得国家、学校、教师、家长、学生以及 社会舆论的共同合法化, 正确认识惩戒的合理性、必 要性和价值性。[15]在互联网时代, 可以借助信息技 术，建设一批适宜传播的“教育惩戒”在线教育教学资 源, 帮助家庭对教育惩戒持有理性的认识和态度。同 时, 家庭教育惩戒要有情有理, 规范家长的惩戒方式, 防止因爱学生而伤学生的悲剧上演, 即使是对犯错误 的孩子进行惩戒, 也应该是充满爱的惩戒, 在惩戒的 时候动之以情, 晓之以理, 让孩子心服口服, 真正地 认识错误、改正错误。尤其是针对一些特殊问题, 教 师实施教育惩戒后的效果并未达到预期目标, 这就需 要积极与家长沟通, 让家庭教育惩戒成为学校教育惩 戒的补充和延续, 使得教育惩戒真正贴近学生、贴切 生活。

\section{4. 多元协同育人，优化社会教育惩戒}

对于不同类型、性质的过失要采用不同的惩戒方 式, 也要由不同的教育惩戒主体来执行, 这样才可以 真正做到具体问题具体分析。对在课堂教学中产生的 轻微问题在学校就可以解决; 需要家庭教育惩戒共同 完成的, 则要共同商讨协作方案。但对于危害社会或 者严重扰乱秩序等情节严重的错误或者犯罪行为, 则 需要社会专门惩戒主体来执行。未成年人社会教育惩 戒机构将法纪教育与立德树人相统一, 进一步完善惩 戒形式和内容, 针对错误产生的原因、出现的情形等, 开展更有针对性的惩戒和教育, 实现学校教育惩戒、 家庭教育惩戒和社会教育惩戒的相互补充和协作, 促 进学生健康成长和全面发展。

\section{4. 结论}

教育惩戒是教育教学的重要手段和形式, 也是教 师教学管理的权利和责任。针对教育惩戒实施标准的 界定较为模糊、教师专业发展水平有待提高、教育惩 戒的协同不够完善、教育惩戒实施效果的评估难以全 面衡量等困境, 为避免因教师实施教育惩戒过程中带 有主观性和随意性, 给学生带来的伤害, 我们应对学 生的违规违纪行为分层分类, 借助“教育戏剧”情景式 教学和教育惩戒在线教育资源, 进一步明确惩戒标准, 规范学校教育惩戒, 推动教师发展, 提升教育惩戒水 平, 完善家庭责任, 协同家庭教育惩戒, 多元协同育 人, 优化社会教育惩戒, 构建多元协同的一体化教育 惩戒体系机制, 提升教育惩戒的系统性, 构建新时代 一体化育人大格局。

\section{项目基金}

本文为全国教育科学 “十三五”规划课题 (BEA200116) 、江苏大学教学改革研究课题 （2019JGZD020）的阶段性成果之一。

\section{REFERENCES}

[1] Chen,Z.L.(2020)Ci Hai.Shanghai dictionaries press, Shanghai.

[2] Wang,Y., Zhang,Z.M.Fang,L.(1989)Punishment and Responsibility.Huaxia Publishing House,Beijing.

[3] Gu,M.Y.(1990)The Dictionary of Education. Shanghai Education Press,Shanghai.

[4] LI,X.J.(2005)Review of Education Disciplinary Research.Forum on Contemporary Education, (22):31-32.

[5] Ministry of Education,2020. Disciplinary Rules for Primary and Secondary Education (Trial). http://www.moe.gov.cn/srcsite/A02/s5911/moe_6 21/202012/t20201228_507882.html.

[6] Ministry of Education,2020. Disciplinary Rules for Primary and Secondary Education (Trial). http://www.moe.gov.cn/srcsite/A02/s5911/moe_6 21/202012/t20201228 507882.html.

[7] Tencent net.2021.After the Second-year Junior High School Students Were Punished for Violation of Discipline and Died, in Addition to Accountability, We Should Also Reflect on How to Give Children Frustration Education. https://xw.qq.com/amphtml/20210414A09I2700.

[8] Xia,L.Y.,Qu,T.H.The Opportunities, Challenges and Development Strategies of Modernization of Teacher Education of Russia in the New Century.Modern Education Management, (07):62-67.

[9] Wang,Y.,Zhang,Z.M.,Fang,L.(1989)Punishment and Responsibility.Huaxia Publishing House,Beijing.

[10] Xinhua News Agency,2020. Law of the People's Republic of China on the Protection of Minors. http://www.gov.cn/xinwen/202010/18/content_5552113.htm.

[11] Li,P.H., Cao, H., Li,X.B.(2011) Study on Property of Teachers Disciplinary Power based on the Power and Right. Meitan Higher Education,29 (05): $80-82+104$.

[12] Liu.C.S., Yang.M.Z.(1981)The Theory of Communism Education. People's Education Press, Beijing. 
[13] Chen.W.Y. The Inner Significance of Teachers'Professional Development in the Context of Educational Modernization The Inner Significance of Teachers'Professional Development in the Context of Educational Modernization. Theory and Practice of Education ,(40-14):38-40.

[14] Zhang.C.My View of Punishment Education.wen jiao zi liao, (05): 136+135.

[15] Zhu,L.Z.(2021)Punishment for Education: from punishment to education punishment. Contemporary Educational Science, (04): 40-45. 